\title{
1 Rewiring PBMC responses to prevent CHIKV infection-specific 2 monocyte subset redistribution and cytokine responses
}

4 José Alberto Aguilar-Briseño1,\#a, Mariana Ruiz Silva1, Jill Moser², Mindaugas

5 Pauzuolis ${ }^{1}$, Jolanda M. Smit ${ }^{1}$, Izabela A. Rodenhuis-Zybert ${ }^{{ }^{*}}$

6

7 1Department of Medical Microbiology and Infection Prevention, University of Groningen 8 and University Medical Center Groningen, 9700 RB Groningen, The Netherlands

9 2Departments of Critical Care, Pathology \& Medical Biology, Medical Biology section, 10 University Medical Center Groningen, University of Groningen, 9700 RB Groningen, The 11 Netherlands

12 \#aCurrent Address: Department of Microbiology and Immunology, University of Iowa, 13 Iowa City 52242, IA, United States of America

16 *Corresponding author:

17 E-mail: i.a.rodenhuis-zybert@umcg.nl 


\section{Abstract}

20 Infection with the mosquito-borne Chikungunya virus (CHIKV) causes acute or chronic

21 arthritis in humans. Inflammatory responses mediated by monocytes, the primary target

22 cells of CHIKV infection in the blood, are considered to play an important role in CHIKV

23 pathogenesis. A recent study revealed that the acute phase of CHIKV infection is

24 characterized by a monocyte-driven response, with an expansion of the intermediate

25 monocyte (IM) subset. In this study, we adopted a previously established in vitro model

26 of CHIKV infection in peripheral blood mononuclear cells, to elucidate the mechanism and

27 relevance of IM expansion in CHIKV replication and associated inflammatory responses.

28 Our data show that infectious but not replication-incompetent CHIKV increases the

29 frequency of IM and to a lesser extent, non-classical (NM) monocytes while reducing the

30 number of classical monocytes (CM). The increase of IM or NM frequency coincided with

31 the activation of inflammatory response and occurred in the absence of lymphocytes

32 implying that monocyte-derived cues are sufficient to drive this effect. Importantly,

33 priming of monocytes with LPS prevented expansion of IM and NM but had no effect on

34 viral replication. It did however alter CHIKV-induced cytokine signature. Taken together,

35 our data delineate the role of IM in CHIKV infection-specific innate immune responses

36 and provide insight for the development of therapeutic strategies that may focus on

37 rewiring monocyte immune responses to prevent CHIKV-mediated arthralgia and

38 arthritis. 


\section{Introduction}

40 Over the last decade, chikungunya virus (CHIKV) infection has affected millions of people

41 worldwide[1]. The vast majority of infected patients experience a febrile illness with

42 painful joints. A substantial number of these individuals, however, develop persistent 43 arthritis-like manifestations (12-49\%)[1]. The exact mechanism underlying CHIKV-

44 mediated pathogenesis is not completely understood, however, clinical and in vivo

45 studies describe local joint inflammation, infiltration of monocytes into the synovial

46 cavity and bone resorption due to increased osteoclast activity[1-3]. Monocytes

47 represent important cellular targets of CHIKV replication in the blood[4,5], and tissue

48 infiltrating monocytes and tissue-resident macrophages have been postulated to act as a

49 vehicles and a reservoir for chronic CHIKV RNA/antigens, respectively [6,7].

50 As innate sentinels of their host, blood monocytes sense invading pathogens and

51 orchestrate innate immune responses to contain their spread as well as setting the scene

52 for the activation of adaptive immune responses[8]. Via a set of pathogen recognition

53 receptors (PRRs), monocytes are able to detect a variety of complex pathogen associated

54 molecular patterns (PAMPS). Engagement of PRRs triggers several cellular signaling

55 cascades resulting in cell differentiation, release of inflammatory mediators and/or

56 programmed cell death[8-10].

57 Monocytes can be divided into three subsets based on CD14 and CD16 surface 58 expression: classical (CD14++CD16-, CM), intermediate (CD14++CD16+, IM) and non59 classical (CD14+CD16++, NM)[11-14]. CM are equipped with a set of PRRs and scavenger

60 receptors, recognizing PAMPS thereby removing microorganisms, lipids, and dying cells

61 via phagocytosis. CM also produce a large variety of effector molecules such as cytokines,

62 myeloperoxidase and superoxide, and play a key role in the initiation of 
inflammation[15]. IM, also known as inflammatory monocytes, selectively traffic to sites

64 of inflammation where they produce inflammatory mediators thereby contributing to

65 local and systemic inflammation[16,17]. IM have the capacity to infiltrate into tissues

66 where they differentiate into inflammatory macrophages and remove PAMPs and cell

67 debris from the microenvironment[18]. NM or patrolling monocytes function as

68 guardians of the vasculature screening for PAMPS. Once activated, they differentiate into

69 anti-inflammatory macrophages to repair damaged tissues[19].

70 During homeostasis, circulating monocytes have a half-life of about one to three days. CM

71 represent the most prevalent fraction (80-90\%) of blood monocytes followed by IM (5\%) and NM (2-5\%). However, stress caused by infection or tissue damage can lead to changes in monocytes viability, subset redistribution, and their effector functions. For instance, viral infections are usually associated with an increase in the frequency of IM. Yet, depending on the etiologic agent, the frequency of CM and NM varies considerably[1522]. Indeed, in vitro engagement of innate receptors by for example TLR7/8 agonists has been shown to cause expansion of IM while TLR4 ligand LPS has been shown to further increase the frequency of $\mathrm{CM}[20]$. The pathogen-tailored responses of the monocyte population are thus likely a result of specific combinations of PAMPs and their respective PRRs, however, the underlying mechanisms remain elusive.

Michlmayr and colleagues recently compared the systemic immune signature of CHIKVinfected individuals in an acute and the convalescent stages of the infection[23]. The ex vivo analysis of PBMCs demonstrated that increased IM frequencies are associated with the induction of inflammation in the acute phase CHIKV infection. Moreover, detection of CHIKV E1 antigen primarily in IM suggested that these cells may also contribute to virus replication [23]. The mechanism and the impact of IM increase on viral replication and 
associated inflammatory responses remain however elusive. Here, we address these using an in vitro model of acute CHIKV infection in primary blood mononuclear cells. By rewiring monocyte responses with different TLR ligands prior to CHIKV exposure, we delineate the role of IM in virus replication and innate responses to CHIKV infection.

\section{Results}

\section{Active CHIKV infection stimulates expansion of intermediate monocytes}

To elucidate the mechanism of monocyte shift during CHIKV infection, we first assessed the contribution of CHIKV particle sensing and/or infection to monocyte subset distribution. We studied monocytes in the context of PBMCs as other cell subsets have previously been shown to be important for the function of monocytes during CHIKV infection[4,5]. PBMCs were isolated from seven donors, as described previously[4] and infected with CHIKV strain LR2006 OPY1 (from hereon called CHIKV) at (multiplicity of infection) MOI 10. The UV-inactivated (UV-CHIKV) was used to evaluate the importance of viral replication in monocytes subset redistribution. At $48 \mathrm{hpi}$, single cell, live monocytes were analyzed on the basis of CD14 and CD16 expression and gated as CD14 and/or CD16 positive cells using the corresponding isotype controls (Fig 1A). The culture of PBMCs in mock conditions (Fig 1B) altered the baseline distribution of monocyte subsets in freshly isolated PBMC[21] in which CM represented the most abundant subtype followed by IM and NM (S1 Fig). Importantly however, in line with the ex vivo findings[23] CHIKV infection induced a significant, approximately 2-fold expansion of IM and NM frequencies and reduction of CM when compared to the mock condition (Fig $1 \mathrm{~B}$ and 1C). No significant changes were observed with UV-CHIKV when compared to the mock which suggests that the increase in IM frequency is driven by CHIKV replication. In contrast to CHIKV, exposure of PBMC to LPS (TLR4/CD14 agonist) led to enrichment of 
111 CM and almost complete depletion of IM corroborating previous findings[20] (Fig 1D).

112 Furthermore, TLR7/8 agonist R848 and TLR2 agonist PAM3CSK4, also increased the

113 frequency of CM (Fig 1D) rather than that of IM as observed by Kwissa et al[20].

114 Importantly, the increase in IM frequency following CHIKV infection was not the result of

115 a replication-mediated cytopathic effect in PBMCs (S2A, S2B and S2C Fig). In fact, only

116 replication-incompetent UV-CHIKV moderately decreased PBMCs viability (S2A Fig).

117 However, when gated on specific cellular populations, neither CHIVK or UV-CHIKV had

118 an effect on the viability of monocytes and lymphocytes (S2B and S2C Fig). In fact, only

119 LPS showed a considerable cytopathic effect on monocytes but not lymphocytes, as

120 compared to mock (S2B and S2C Fig). Furthermore, the redistribution of monocyte

121 subsets following CHIKV infection was largely independent of cues from other cells since

122 the effect was sustained in PBMCs depleted of T and B lymphocytes, NK and DCs (S3 Fig).

A

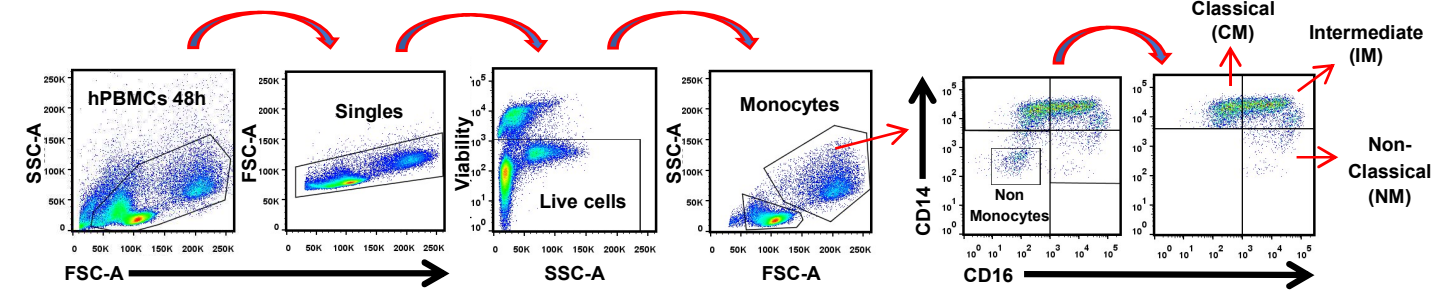

126

127

128

129

130

131

132
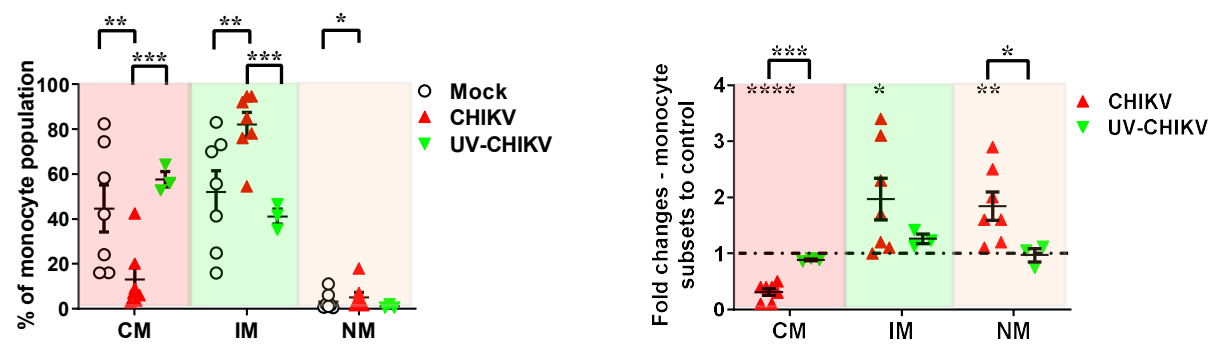

D

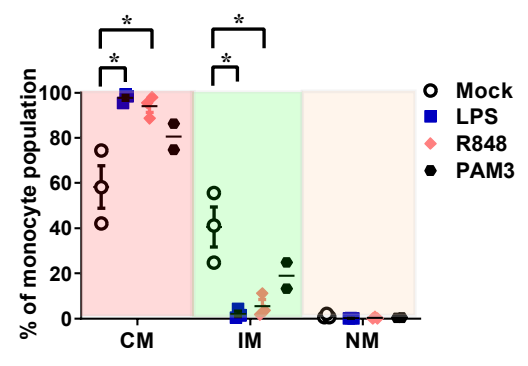


133 Fig 1. CHIKV infection increases the frequency of intermediate monocytes. (A-D) PBMCs

134 from healthy donors (n=3-7) were either infected with CHIKV-LR at MOI of $10(n=7)$ or its UV-

135 inactivated equivalent (UV-CHIKV) (n=3), or treated with LPS (100 ng/mL, n=3), R848 (2 $\mu \mathrm{g} / \mathrm{mL}$,

$136 \mathrm{n}=3$ ), PAM3CSK4 (PAM3, 50ng/mL, n=2) for 48hpi. (A) Gating strategy to define monocyte

137 subsets. (B) Frequencies of monocyte subsets after infection with CHIKV depicted as percentages

138 or (C) as fold changes relative to the mock. (D) Frequencies of monocyte subsets after treatment

139 with TLR agonists. CM: classical monocytes, IM: intermediate monocytes and NM: non-classical

140 monocytes. Bar represents mean \pm SEM. P values were obtained by paired (D) or unpaired (B and

141 C) one-tailed t test. $\left({ }^{*} \mathrm{P}<0.05,{ }^{* *} \mathrm{P}<0.01,{ }^{* * *} \mathrm{P}<0.001,{ }^{* * * *} \mathrm{P}<0.0001\right)$.

142 CHIKV infection-mediated shift in IM coincides with induction of specific 143 inflammatory responses

144 We next assessed if monocyte redistribution influenced CHIKV-infection specific cytokine 145 signatures (Fig 2A). Initial assessment of gene expression analysis of several 146 inflammatory cytokines from 2 donors showed that replication-competent CHIKV but not 147 the UV-inactivated virus increased the mRNA levels of $I L-6$ and $I F N-\beta$ (Fig $2 B$ ). On the 148 other hand, UV-CHIKV increased the expression of $I L-8$ mRNA by circa 2-fold while 149 replication-competent CHIKV reduced its expression (Fig 2B). Similar mRNA expression 150 signatures were found at 24 hpi for $I L-6, I L-8$ and $I F N-\beta$, but not TNF- $\alpha$ (S4 Fig). 151 Subsequent multiplex analysis of cytokines released from infected PBMCs confirmed our 152 gene expression findings for IL-6 and IFN- $\beta$ (Fig 2C). However, no TNF- $\alpha$ production was 153 detected in the supernatants of infected PBMCs, suggesting that either the observed 154 increase in TNF- $\alpha$ mRNA levels did not result in TNF- $\alpha$ production or it is produced later 155 than 48hpi. Unfortunately, IL-8 levels present in all conditions reached the upper limit of 156 quantification, thereby preventing verification of the CHIKV-specific reduction of IL-8 157 mRNA. Furthermore, CHIKV but not UV-CHIKV, led to the increase of inflammatory 
mediators such as IL-10, IP-10 and IFN- $\alpha 2$ (Fig 2C). There was no effect on the gene expression levels of MAVS or IRF3 (S5 Fig), which might suggest that the observed IFN type I production following CHIKV infection was independent of RIG-I/MDA5-mediated sensing of dsRNA[24,25]. We detected no changes in mock levels of TNF- $\alpha$, IL-1 $\beta$, IL12p70, GM-CSF, IFN- $\gamma$, IFN- $\lambda 1$ and IFN- $\lambda 2 / 3$ following exposure to (UV-)CHIKV.

Next, we sought to elucidate if CHIKV-mediated responses are specifically associated with an increase in IM and NM frequencies. To this end, we first evaluated the expression levels of the same inflammatory mediators as above but now following the exposure of PBMCs to LPS, which in contrast to CHIKV, increased the frequency of CM (Fig 1D). To avoid a possible bias in expression levels of inflammatory mediators caused by a moderate, albeit consistent, cytopathic effect of LPS on PBMCs at 48hpi (S2 Fig), we evaluated LPS-specific responses at both at $24 \mathrm{hpi}$ and 48hpi (Fig 3 and S6 Fig). Importantly, in line with data from 48hpi, exposure of PBMC to LPS for $24 \mathrm{hpi}$ (hereafter referred to as priming) increased CM frequency and concurrently virtually depleted IM and NM subsets (Fig 3). At the same time, priming had no effect on baseline (mock) mRNA expression levels of TNF- $\alpha$ or $I F N-\beta$, but induced marked 30- and 1000-fold increase in transcription of $I L-8$ and IL-6 genes, respectively (Fig 3 and S6 Fig). Thus, LPS-mediated shift of monocytes subsets and concurrent inflammatory responses were markedly different from those initiated upon CHIKV infection alone. 
177

178

179

180

181

182

183

184

185

186

187

188

189

190

191

192

193

194
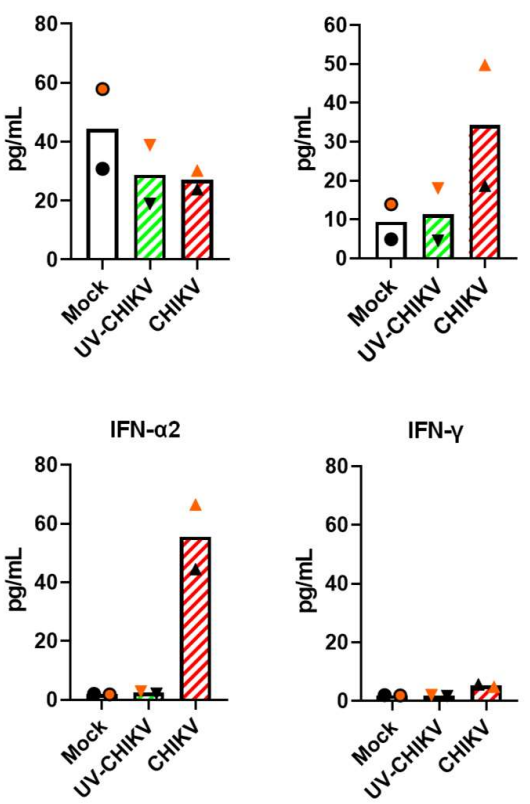

B
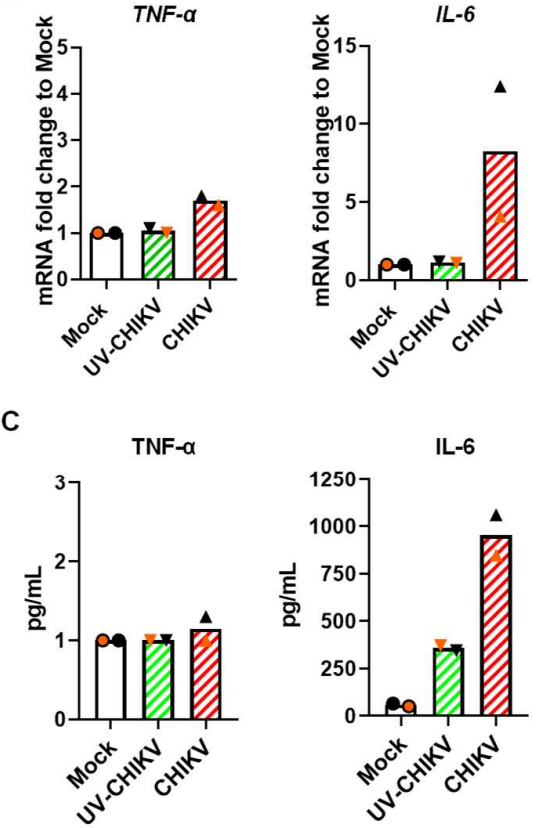

IL-1 $\beta$

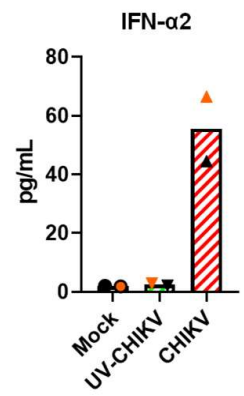

Gene expression analysis -Cytokine production
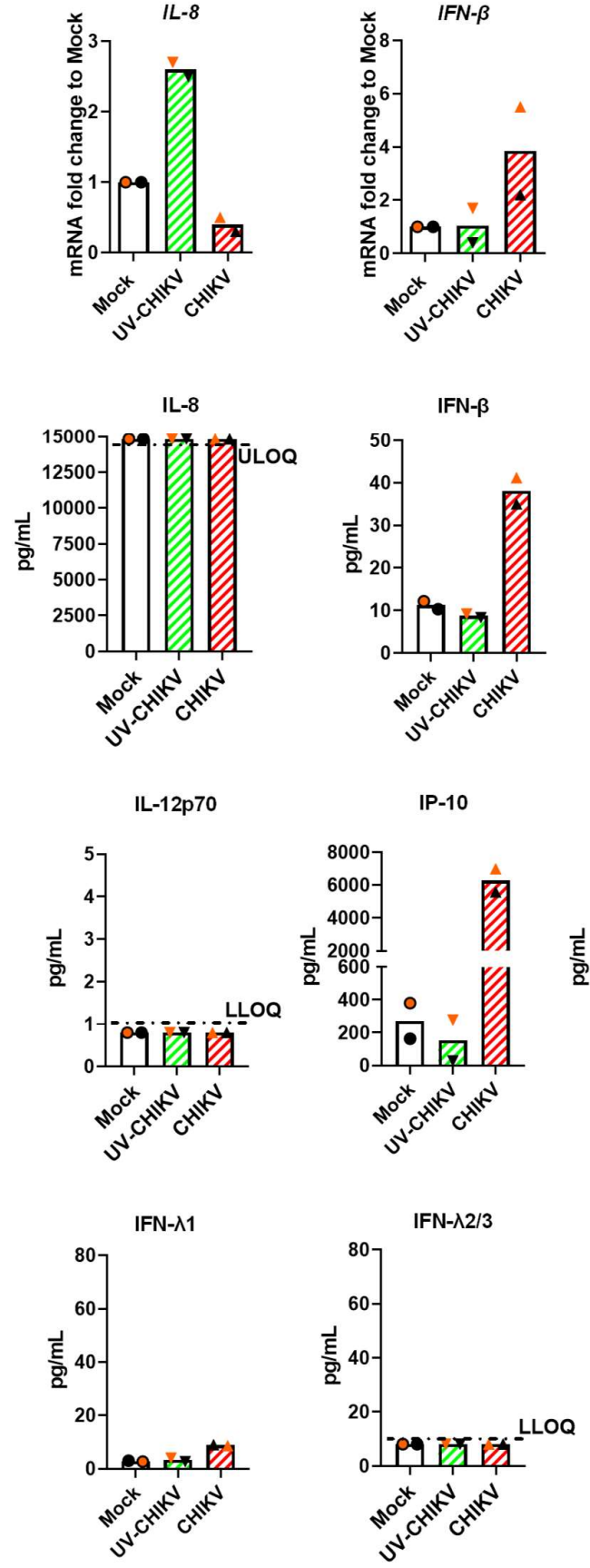
196 Fig 2. Differential immune responses induced by CHIKV and UV-CHIKV. (A) PBMCs from

197 healthy donors $(\mathrm{n}=2$, orange= donor 1 , black= donor 2$)$ were (mock)-treated with LPS (600 $198 \mathrm{ng} / \mathrm{mL}$ ), CHIKV-LR at MOI of 10 or its UV- inactivated equivalent (UV-CHIKV), for 48h. (B) Fold 199 changes in gene expression of TNF- $\alpha, I L-6, I L-8$ and $I F N-\beta$ relative to the respective mock. Mock $20048 \mathrm{~h}$ was set as a reference sample. YWHAZ was set as a reference gene. (C) Concentrations of 201 released cytokines in picograms per milliliter $(\mathrm{pg} / \mathrm{mL})$ were determined using LegendPlex. ULOQ 202 and LLOQ (horizontal dotted line) indicate upper and lower limit of quantification, respectively.

A

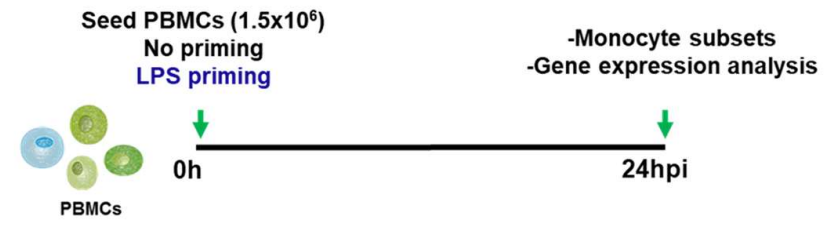

B

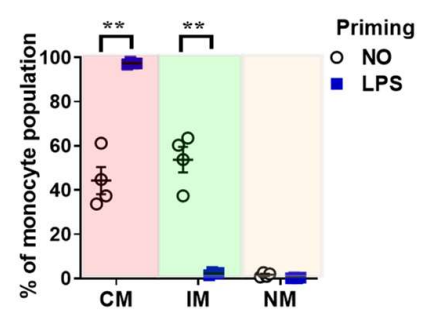

C

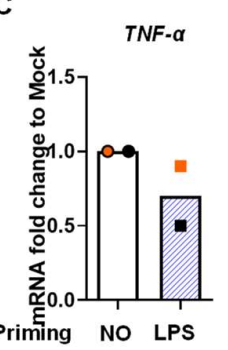

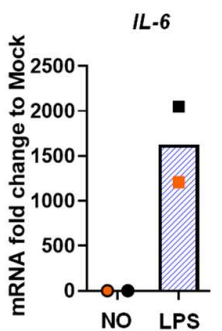

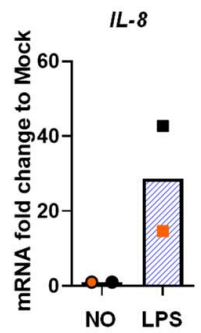

Fig 3. Effect of LPS treatment in cytokines, gene expression and monocyte subsets

distribution. (A) PBMCs from healthy donors $(n=2-4)$ were (mock)-treated with LPS (600 ng/Ml) for 24h. (B) Monocyte subsets distribution as determined by flow cytometry. (C) Fold changes in gene expression of $T N F-\alpha, I L-6, I L-8$ and $I F N-\beta$ relative to the respective mock. Mock $24 \mathrm{~h}$ was set as a reference sample. $Y W H A Z$ was used as a reference gene. Orange symbol=donor mean \pm SEM. P values were obtained by paired one-tailed $t$ test; ${ }^{* *} \mathrm{P}<0.01$. 
217 Preventing IM expansion alters immune responses but has no effect on CHIKV 218 production

219 Expansion of a particular monocyte subset often corresponds to the cellular tropism of 220 the virus, as it has been shown for HIV-1, hepatitis C virus (HCV) and Zika virus 221 (ZIKV)[22,26-29]. Since in the acute phase of infection, CHIKV E1 antigen was found 222 primarily in human IM, we first verified that this is also the case in our in vitro system. 223 Indeed, and as expected here considering the prevalence of IM following the infection, 224 CHIKV Ag was present primarily within IM population of PBMCs exposed to replicationcompetent CHIKV (S7 Fig). Consequently, we next hypothesized that if CHIKV infection leads to the expansion of more susceptible monocyte subsets, priming of the cells with LPS prior to infection would reduce viral replication and likely alter CHIKV-specific immune responses (Fig 4A). Priming PBMCs with LPS prevented subsequent CHIKV infection-mediated increase in IM pool and instead increased CM frequency (Fig 4B). Despite the profound effect on the distribution of monocytes subsets, priming did not influence infectious virus production (Fig 4C). Thus, IM are not more susceptible and/or permissive to CHIKV infection than CM or NM. Rather, due to the substantial increase in the IM population, the likelihood of virus infecting this monocyte population is higher. Priming did however alter the response of PBMC to both UV-CHIKV and infectious virus (Fig 4D). The levels of cytokines including IL-6, IL-8, IFN- $\beta$, IL-1 $\beta$, GM-CSF and IFN- $\gamma$ were higher in primed and CHIKV-challenged samples when compared to their non-primed counterparts. In contrast, IP-10 and IFN- $\alpha 2$ levels were similar in primed and nonchallenged (mock) conditions, indicating that priming overruled and/or prevented 
242 and IFN- $\gamma$ in response to challenge with UV-CHIKV. The priming had no effect on the

243 levels of IFN- $\lambda 1$ and IFN- $\lambda 2$ in any condition tested. Altogether, these data suggest that

244 LPS priming alters both monocyte subset distribution and immune responses to CHIKV

245 without affecting virus replication (Fig 5).

246

247

248

249

250

251

252

253

254

255

256

257

258

259

260

261

262

A
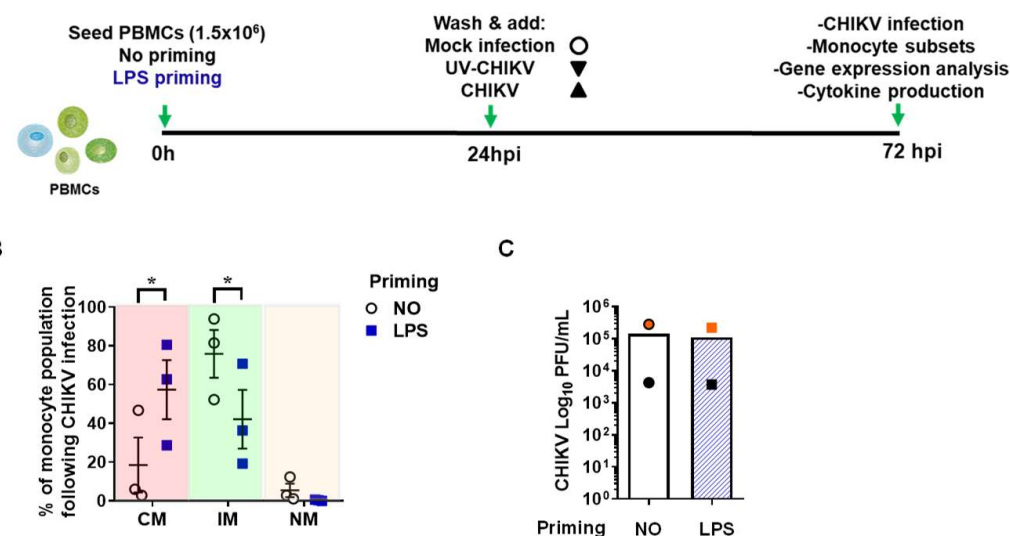

D
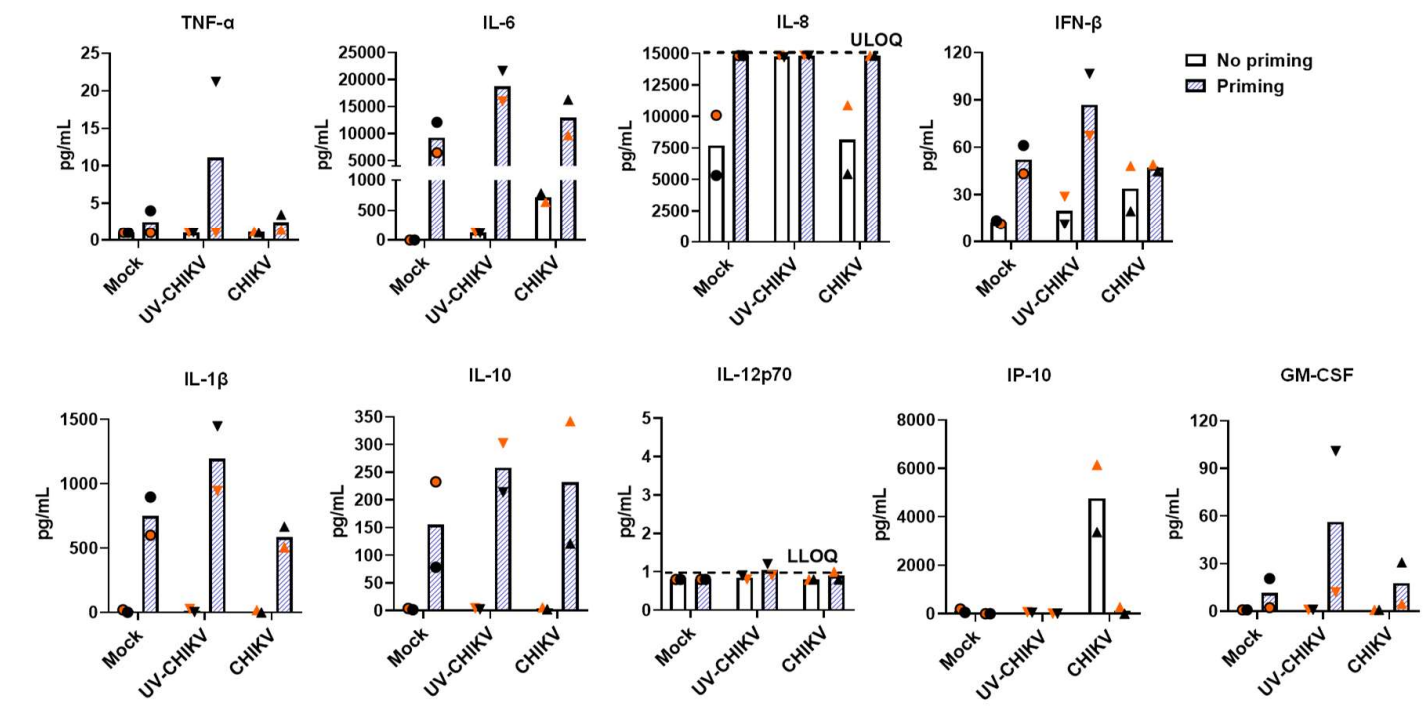

263

264

265

266

267
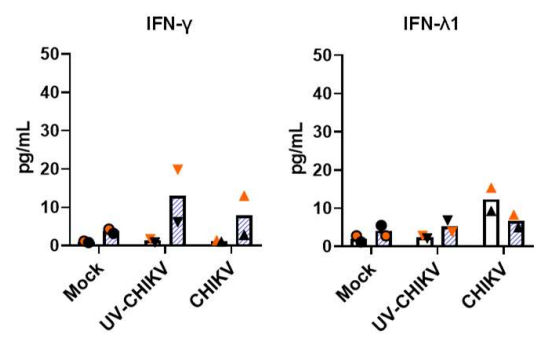
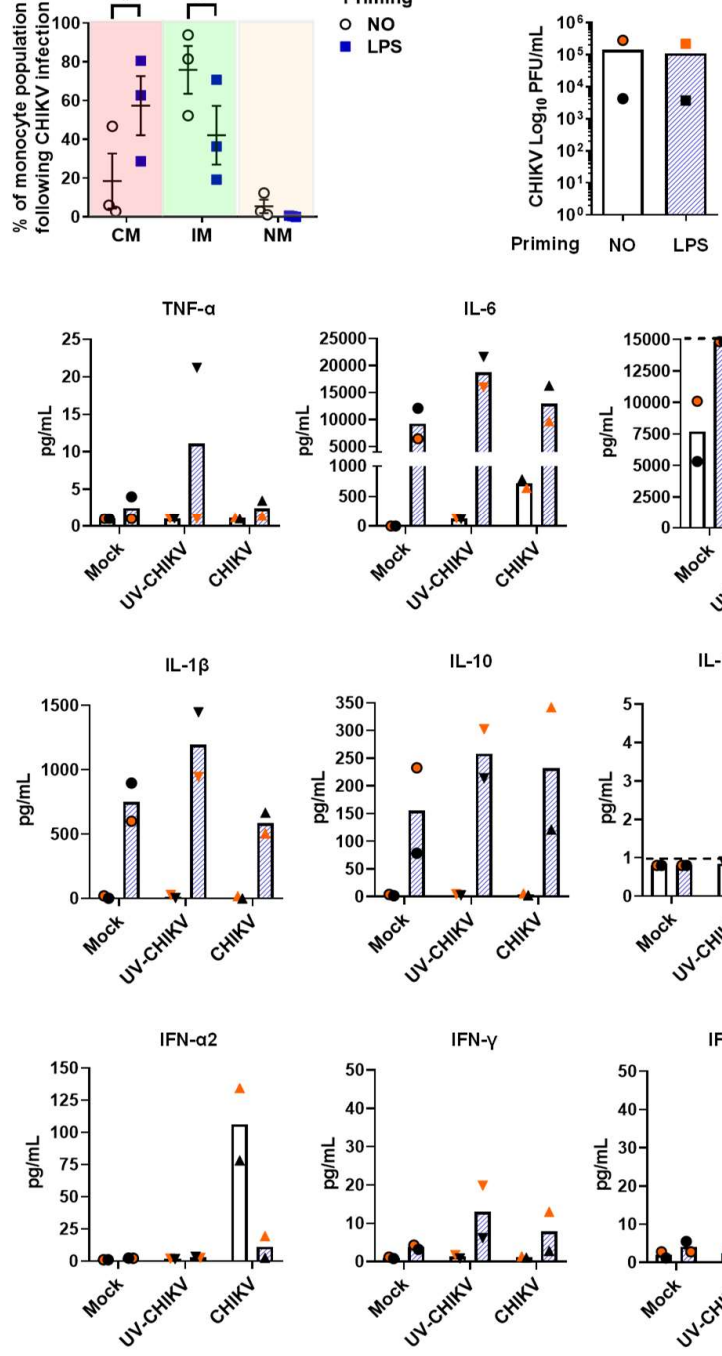

Fig 4. Effect of LPS priming on the infection and innate immune responses following CHIKV

infection. (A) PBMCs from healthy donors (n=2-3) were (mock)-treated with LPS (600 ng/mL) 
270 for $24 \mathrm{~h}$ and then (mock)-treated with LPS $(600 \mathrm{ng} / \mathrm{mL})$, CHIKV-LR at MOI of 10 or its UV-

271 inactivated equivalent (UV-CHIKV). (B) Frequencies of monocyte subsets after infection with

272 CHIKV. (C) Plaque forming units (PFU) were measured by plaque assay. (D) Concentrations of

273 released cytokines in picograms per milliliter $(\mathrm{pg} / \mathrm{mL})$ were determined using LegendPlex. ULOQ

274 and LLOQ (horizontal dotted line) indicate upper and lower limit of quantification, respectively.

275 Orange symbol= donor 1 , black symbol= donor 2 . CM: classical monocytes, IM: intermediate

276 monocytes and NM: non-classical monocytes. Bar represents mean \pm SEM. P values were obtained

277 by paired one-tailed t test. $(* \mathrm{P}<0.05)$.

Fig 5. Summary of the results. CHIKV infection of human PBMCs induces an infection-specific innate immune response hallmarked by the increased frequency of intermediate monocytes (IM) and the production of inflammatory mediators such as IL-6, IP-10, IFN- $\alpha$ and INF- $\beta$. Priming PBMCs with LPS prevents CHIKV infection-induced monocyte subset redistribution and cytokine responses without altering CHIKV replication. Figure created with BioRender.com

\section{Discussion}

290 Here, we used a previously established in vitro model of CHIKV infection in peripheral blood mononuclear cells to elucidate the mechanism and effect of IM expansion in the acute phase of CHIKV infection[23] on virus replication and associated inflammatory 
responses. Our findings indicate that the increase in IM population is dependent on

294 CHIKV replication and occurs irrespectively of cues from other leukocytes. Furthermore,

295 the redistribution of monocytes subsets coincided with the presence of CHIKV Ag in IM

296 and infection-mediated inflammatory responses. By rewiring PBMCs responses with LPS,

297 we could prevent CHIKV infection-specific monocyte subset redistribution and cytokine

298 responses without altering virus replication.

299 An increase in IM has been also been reported during the course of several other chronic 300 inflammatory diseases such as rheumatoid arthritis (RA)[30-33]. This indicates that the imbalance in the frequency of monocytes contributes to persistent and exacerbated inflammation. Indeed, Chimen et al.,[34] suggested that during inflammation, NM and IM transmigrate more rapidly across the endothelium where they produce high levels of

TNF- $\alpha$ thereby promoting neutrophil recruitment into the inflamed tissue. In contrast,

CM migrate slower than CD16+ monocytes and upon arrival at the site of inflammation decrease neutrophil recruitment by producing IL-6[34]. Consequently, factors preventing expansion of IM by either triggering CM phenotype by LPS priming as presented here, or depleting IM by glucocorticoid therapy, could be applied to decrease inflammation in diseases hallmarked by an expansion of CD16+ monocytes[35]. Importantly, the expansion of IM and NM and concurrent reduction of CM frequency described here in our in vitro model of CHIKV infection, underscores the ability of CM to differentiate into IM and finally to NM as suggested by previous in vivo studies[13,36-39]. Importantly, as life spans and migratory function differ between the monocytes subsets[13,17], future studies should elucidate how quickly conversion occurs, and how long each of these subsets remain in circulation following CHIKV infection before 316 infiltrating synovial tissue. Identifying the mechanisms governing the regulation of 317 monocyte differentiation as well as its kinetics and function will be crucial to better 
understand their role in the pathogenesis of CHIKV-mediated arthritis that will facilitate the development of immunomodulatory therapies.

Expansion of a particular monocyte subset has been shown to correspond to the cellular tropism of many viruses. For instance, HIV-1 causes expansion of IM and NM and these cells are more permissive to the virus than CM, mainly because they express the entry receptors for HIV-1, CCR5 and CD4[22,26]. Likewise, hepatitis C virus (HCV) triggers an increase of IM and NM and preferentially infects these subsets as they express high levels of the virus cell entry receptor CD81[27,28]. Zika virus (ZIKV) causes expansion of the IM and these cells are also the most susceptible monocytes to infection[29]. While we were working on this manuscript, Michlmayr and colleagues reported that CHIKV Ag could be found primarily in IM of infected patients[23]. Based on these observations we hypothesized that altering the numbers of IM would have an effect on virus replication. To investigate this we took advantage of findings which showed opposing effects of TLR4 and TLR2/7/8 ligands[20] on CM and IM frequencies. Importantly, however, our study demonstrates that although CHIKV virus antigen is found in IM, lack of this particular subset and predominance of CM in LPS primed PBMC did not affect virus replication. This indicates that in the case of CHIKV, IM expansion does not represent a pro-viral mechanism, rather an innate response of the host to a particular set of viral PAMPs. Moreover, since the increase in IM population occurred independently of lymphocytes, PRRs expressed on monocytes are likely to govern the mechanisms underlying monocyte differentiation. Finally, given that UV-inactivated CHIKV failed to trigger IM expansion, it is likely that RNA sensing TLRs such as TLR3/7/8 [3,40-42] and/or RLRs[43] play an important role in the process. 
341 Counteracting pro-inflammatory mediators coupled with a simultaneous boost of

342 antiviral responses might be the most successful approach to achieve balanced innate

343 responses that will favour virus containment without causing aberrant pathologies.

344 Accordingly, rewiring inflammatory responses without reducing virus replication, as

345 observed here in LPS-primed PBMCs, is arguably a suboptimal strategy to mitigate CHIKV

346 disease. Interestingly, however, rewiring immune responses in CHIKV-infected mice with

347 bindarit, an anti-inflammatory drug blocking inter alia MCP-1 production[44], reduced

348 joint swelling and prevented bone erosion despite having no effect on viremia levels[45].

349 Thus, mitigation of disease may be achieved without decreasing viral replication. Indeed,

350 there is no consensus regarding the correlation between peak viremia CHIKV titers and

351 disease progression[23,46]. Since our study did not assess the effect of LPS priming on

352 CHIKV pathogenesis, we cannot, nor do we intend to, equate the effect of LPS found in

353 PBMCs with that found in vivo with MCP-1 inhibitor. However, TLR4-mediated reduction

354 of MCP-1 expression in monocytes is among several identified responses in LPS-treated

355 PBMCs[47]. Based on these observations, it would be worthwhile to assess the effect of

356 LPS on CHIKV pathogenesis in vivo. Moreover, given that increased levels of LPS in the

357 blood have been found in several inflammatory diseases [48-50], such studies will inform

358 us how co-microbial infections and/or how microbial translocation will influence host

359 responses to CHIKV and ultimately disease progression. In addition, they are imperative

360 to understanding whether pharmacological targeting of inflammatory mechanisms early

361 in infection could serve as a strategy to prevent CHIKV-mediated arthralgia and arthritis,

362 possibly, even without the necessity to decrease viral replication.

363 In summary, our study provides important insights into the mechanism and role of IM

364 expansion in CHIKV replication and infection-mediated immune responses. Further 
studies are required to identify receptors involved in monocyte differentiation during

366 CHIKV infection and validate their function in disease progression.

\section{Methods}

\section{Cells}

PBMCs were isolated by standard density gradient centrifugation procedures with Ficoll-

371 from healthy volunteers, in line with the declaration on Helsinki (Sanquin Bloodbank,

372 Groningen, the Netherlands). The PBMCs were cryopreserved at $-196{ }^{\circ} \mathrm{C}$. Vero-E6 and

373 Vero-WHO were cultured in DMEM supplemented with FBS, penicillin (100 U/mL),

374 streptomycin $(100 \mu \mathrm{g} / \mathrm{mL})$, HEPES $(10 \mathrm{mM})$ and glutamine $(200 \mathrm{mM})$. All of the cell lines

375 used were tested negative for the presence of Mycoplasma spp. using a commercial

376 functional method (Lonza, the Netherlands) and/or in-house qPCR assay adapted from

377 Baronti et al.,[51].

\section{Monocyte enrichment}

Monocytes were isolated from thawed PBMCs using the MagniSort ${ }^{\mathrm{TM}}$ human panmonocyte enrichment kit (eBioscience). Briefly, a single-cell suspension containing $1 \times$ $10^{8}$ PBMCs per mL of cell separation buffer (PBS, 3\% FBS, 10mM EDTA) was prepared.

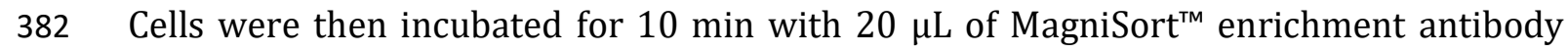
cocktail per $100 \mu \mathrm{L}$ of cell suspension. Cells were washed and resuspended in separation buffer. Then $10 \mu \mathrm{L}$ of MagniSort ${ }^{\mathrm{TM}}$ negative selection beads were added per $100 \mu \mathrm{L}$ of cell 
by flow cytometry staining with anti-human CD14 eFluor 450 (clone 613D, eBioscience) and anti-human CD16 APC (clone CB16, eBioscience).

\section{Virus}

CHIKV (La Reunion OPY1) was a gift from A. Merits (University of Tartu, Estonia), and of plaque-forming units (PFU) by standard plaque assay on Vero WHO and the number of genome-equivalent copies (GEc) by quantitative RT-PCR (RT-qPCR), as described previously[53]. Virus inactivation was performed by $1.5 \mathrm{~h}$ incubation of virus aliquots under UVS-28 8-watt Lamp. Inactivation below level of detection $35 \mathrm{PFU} / \mathrm{ml}$ was confirmed using standard plaque assay on Vero-WHO[53]. CHIKV stock used in this study tested negative for Mycoplasma spp. using a commercial functional method (Lonza, the Netherlands) and/or in-house qPCR assay adapted from Baronti et al., [51].

\section{In vitro infections}

PBMCs $\left(1 \times 10^{6}\right.$ cells $\left./ \mathrm{mL}\right)$ were treated with PAM3CSK4 (50 ng/mL, InvivoGen), LPS multiplicity of infection (MOI) of 10 or its UV- inactivated equivalent (UV-CHIKV-LR) for 48h. Monocytes ( $2 \times 10^{5}$ cells) were infected with CHIKV-LR at MOI 10 or its equivalent UV-CHIKV-LR for 48h. To evaluate the effect of the LPS priming on CHIKV infection, PBMCs were (mock)-primed with LPS for $24 \mathrm{~h}$ and then (mock)-treated with LPS or infected with CHIKV-LR at MOI 10 for $48 \mathrm{~h}$ or its equivalent UV-CHIKV-LR for $48 \mathrm{~h}$. For viral production, CHIKV-LR infected cells were washed after infection and media replenished $(600 \mu \mathrm{L}), 48 \mathrm{~h}$ later cell-free supernatants were collected and preserved at - 
411 cell lysates were collected at $48 \mathrm{~h}, 24 \mathrm{~h}$ priming and $48 \mathrm{~h}$ post-priming, samples were

412 preserved at $-80^{\circ} \mathrm{C}$ and $-20^{\circ} \mathrm{C}$, respectively.

413 Flow cytometry analysis

414 Surface expression of CD14 and CD16 was measured on PBMCs and enriched monocytes

415 directly after thawing of the cells, after infection with CHIKV or treatment with the 416 agonists but prior to infection and $24 \mathrm{~h}$ and $48 \mathrm{~h}$ post infection/treatment with the 417 agonists. Briefly, PBMCs were stained with Fixable Viability Dye eFluor 780, anti-human 418 CD14 eFluor 450 (clone 613D) and anti-human CD16 APC (clone CB16). All purchased 419 from eBioscience. Samples were measured on a FACSverse flow cytometer (BD 420 Biosciences). Isotype-matched antibodies labelled eFluor 450 (clone P3.6.2.8.1) and APC 421 (clone P3.6.2.8.1), both eBioscience, were used as controls to compare the expression of 422 each marker. To measure the number of infected monocytes, PBMCs were fixed at 48hpi 423 and stained intracellularly using CHIKV E1-specific rabbit antibody (kindly gifted by dr.

424 G. Pijlman from Wageningen University) and secondary chicken anti-rabbit AF647 (Life 425 Technologies). Samples were measured on Aurora flow cytometer (Cytek®). Data were 426 analyzed using the Flowjo software (BD Biosciences).

\section{Gene expression analysis}

428 Total RNA was isolated from PBMCs using a RNeasy Plus Mini Kit (Qiagen, Leusden, The 429 Netherlands), according to the manufacturer's instructions. RNA integrity was checked, 430 cDNA synthesized, and RT-qPCR performed using the ViiA 7 system (Applied 431 Biosystems/ThermoFisher Scientific) as previously described[54]. Assay on demand 432 primers were from Applied Biosystems (Nieuwerkerk aan de IJssel, The Netherlands) 433 included IL-6 (Interleukin-6, Hs00174131_m1), IL-8 (Interleukin-8, Hs00174103_m1), 434 TNF- $\alpha$ (Tumor necrosis factor alpha, Hs00174128_m1), IFN- $\beta$ (Interferon beta, 
435 Hs01077958_s1), IRF3 (IFN regulatory factor 3, Hs01547283_m1) and MAVS

436 (Mitochondrial antiviral signaling protein, Hs00920075_m1). Duplicate real-time PCR

437 analyses were performed for each sample, and the obtained threshold cycle (CT) values

438 were averaged. Gene expression was normalized to the expression of housekeeping gene

439 (YWHAZ, Hs01122445_g1) resulting in the $\Delta$ CT value. The relative mRNA level was

440 calculated by $2^{-\Delta \mathrm{CT}}$.

\section{Multiplex analysis of cytokines and chemokines}

442 Human anti-virus response panel (13-plex, LEGENDplex ${ }^{\mathrm{TM}}$, BioLegend) was used to 443 determine the protein levels of IL-1 $\beta$, TNF- $\alpha$, IL-6, IL-8, IL-10, IL-12p70, IP-10, GM-CSF, $444 \quad$ IFN- $\alpha 2$, IFN- $\beta$, IFN- $\gamma$, IFN- $\lambda 1$ and IFN- $\lambda 2 / 3$. Data were collected using a FACSverse flow 445 cytometer (BD Biosciences) and analyzed with LEGENDplex ${ }^{\mathrm{TM}}$ v8.0 (BioLegend).

\section{Statistical analysis}

447 Data analysis was performed using Prism 6.01 (Graphpad, USA). Data are shown as mean $448 \pm$ SEM. Paired one-tailed t-test or unpaired one-tailed t-test were used to determine 449 statistical significance. In all tests, values of ${ }^{*} \mathrm{p}<0.05,{ }^{* *} \mathrm{p}<0.01,{ }^{* * *} \mathrm{p}<0.001$ and $* * * * \mathrm{p}<0.0001$ were considered significant.

\section{Supporting information}

S1 Fig. Changes in the monocyte subsets distribution due to in vitro culturing. PBMCs from healthy donors $(n=1)$ were cultured for 48 h. Frequencies of monocyte subsets were determined by flow cytometry immediately after thawing and at $48 \mathrm{~h}$ of culture. 
458 (UV-CHIKV) (n=4), for 48h. PBMCs were collected and stained with fixable viability dye. (A)

459 Viability of total PBMCs, (B) monocytes and (C) lymphocytes. Bar represents mean \pm SEM. P

460 values were obtained by unpaired one-tailed test $\left(* \mathrm{P}<0.05\right.$, $\left.{ }^{* *} \mathrm{P}<0.01\right)$.

461 S3 Fig. Active CHIKV infection increases intermediate monocytes' frequency. Monocytes

462 were enriched by negative selection from PBMCs isolated from healthy donors $(n=3)$ and then

463 (mock)- infected with CHIKV-LR at MOI of 10 for 48h. (A) Enrichment efficiency as assessed by

464 flow cytometry (B) Frequencies of monocyte subsets were determined by flow cytometry after

465 infection. CM: classical monocytes, IM: intermediate monocytes and NM: non-classical monocytes.

466 Bar represents mean \pm SEM. P values were obtained by paired one-tailed $t$ test $\left({ }^{*} \mathrm{P}<0.05\right)$.

467 S4 Fig. Differential expression of cytokines after 24h of infection with CHIKV. PBMCs from

468 healthy donors $(\mathrm{n}=2$, orange= donor 1 , black= donor 2$)$ were (mock)-infected with CHIKV-LR at

469 MOI of 10 for $24 \mathrm{~h}$. Fold changes in gene expression of TNF- $\alpha, I L-6, I L-8$ and $I F N-\beta$ relative to the

470 respective mock. Mock 24h was set as a reference sample. YWHAZ was used as a reference gene.

471 S5 Fig. Gene expression levels of $I R F 3$ and $M A V S$ after $24 \mathrm{~h}$ and $48 \mathrm{~h}$ of culture. PBMCs from

472 healthy donors $(n=2$, orange= donor 1 , black= donor 2$)$ were cultured for $24 \mathrm{~h}$ and $48 \mathrm{~h}$. Fold

473 changes in gene expression of IRF3 and MAVS relative to the respective mock. $0 \mathrm{~h}$ was set as a

474 reference sample. $Y W H A Z$ was used as a reference gene.

475 S6 Fig. Differential expression of cytokines induced by LPS. PBMCs from healthy donors (n=2)

476 were (mock)-treated with LPS (100 ng/mL, $\mathrm{n}=2)$ for $48 \mathrm{~h}$. Fold changes in gene expression of TNF-

$477 \alpha, I L-6, I L-8$ and $I F N-\beta$ relative to the respective. Mock $48 \mathrm{~h}$ was set as a reference sample. YWHAZ

478 was used as a reference gene.

479 S7 Fig. CHIKV replicates primarily in IM. PBMCs from healthy donors (n=1) were (mock)480 infected with CHIKV-LR at MOI of 20 or its UV- inactivated equivalent (UV-CHIKV) for $48 \mathrm{~h}$. 481 Frequencies of CHIKV positive cells and monocyte subsets distribution were determined by flow 482 cytometry. 


\section{Acknowledgments}

484 We thank Heidi Ende-Metselaar for her excellent technical assistance. We thank Peter 485 Zwiers (Endothelial Biomedicine \& Vascular Drug Targeting group, UMCG) for his 486 assistance and guidance in the gene expression experiments. We are grateful to Geert

487 Mesander (Flow Cytometry Unit, UMCG) for his technical expertise and guidance in the 488 flow cytometry assays.

\section{Author contributions}

Conceptualization: José Alberto Aguilar Briseño, Mariana Ruiz Silva, Izabela A Rodenhuis-Zybert

Data curation: José Alberto Aguilar Briseño, Mariana Ruiz Silva

Formal analysis: José Alberto Aguilar Briseño, Mariana Ruiz Silva, Izabela A Rodenhuis-Zybert. Zybert.

Investigation: José Alberto Aguilar Briseño, Mariana Ruiz Silva, Mindaugas Pauzuolis.

Methodology: José Alberto Aguilar Briseño, Izabela A Rodenhuis-Zybert.

Project administration: Izabela A Rodenhuis-Zybert.

Resources: Jill Moser, Jolanda M Smit, Izabela A Rodenhuis-Zybert.

Supervision: Izabela A Rodenhuis-Zybert.

Validation: José Alberto Aguilar Briseño, Mariana Ruiz Silva, Izabela A Rodenhuis-Zybert.

Visualization: José Alberto Aguilar Briseño.

503 Writing - original draft: José Alberto Aguilar Briseño, Mariana Ruiz Silva, Izabela A Rodenhuis504 Zybert.

505 Writing - review \& editing: José Alberto Aguilar Briseño, Jill Moser, Jolanda M Smit, Izabela 506 A Rodenhuis-Zybert. 
508 Data Availability Statement: All relevant data are within the manuscript and its 509 supplementary information files.

510 Funding: JAAB was supported by de Cock - Hadders Stichting grant and by CONACyT,

511 Mexico. MRS was supported by de Cock - Hadders Stichting grant. IARZ was supported 512 by Research Grant 2019 from the European Society of Clinical Microbiology and 513 Infectious Diseases (ESCMID). Funding agencies had no role in the experimental design, 514 decision to publish, or preparation of the manuscript.

515 Competing interests: The authors declare that they have no competing interests.

\section{References}

518 1. Dupuis-Maguiraga L, Noret M, Brun S, Le Grand R, Gras G, Roques P. Chikungunya

3. Schwartz O, Albert ML. Biology and pathogenesis of chikungunya virus. Nature Reviews Microbiology. 2010.pp. 491-500. doi:10.1038/nrmicro2368

4. Her Z, Malleret B, Chan M, Ong EKS, Wong S-C, Kwek DJC, et al. Active Infection of Human Blood Monocytes by Chikungunya Virus Triggers an Innate Immune Response. J Immunol. 2010;184: 5903-5913. doi:10.4049/jimmunol.0904181 IA. Mechanism and role of MCP-1 upregulation upon chikungunya virus infection in human peripheral blood mononuclear cells. Sci Rep. 2016;6: 32288. doi:10.1038/srep32288

6. Labadie K, Larcher T, Joubert C, Mannioui A, Delache B, Brochard P, et al. 
Chikungunya disease in nonhuman primates involves long-term viral persistence in macrophages. J Clin Invest. 2010;120: 894-906. doi:10.1172/JCI40104

7. Hoarau J-J, Jaffar Bandjee M-C, Krejbich Trotot P, Das T, Li-Pat-Yuen G, Dassa B, et al. Persistent Chronic Inflammation and Infection by Chikungunya Arthritogenic Alphavirus in Spite of a Robust Host Immune Response. J Immunol. 2010;184: 5914-5927. doi:10.4049/jimmunol.0900255

8. Lauvau G, Loke P, Hohl TM. Monocyte-mediated defense against bacteria, fungi, and parasites. Seminars in Immunology. 2015. pp. 397-409. doi:10.1016/j.smim.2016.03.014

9. Chow J, Franz KM, Kagan JC. PRRs are watching you: Localization of innate sensing and signaling regulators. Virology. 2015;479-480: 104-109. doi:10.1016/j.virol.2015.02.051

10. Gaidt MM, Ebert TS, Chauhan D, Schmidt T, Schmid-Burgk JL, Rapino F, et al. Human Monocytes Engage an Alternative Inflammasome Pathway. Immunity. 2016;44: 833-846. doi:10.1016/j.immuni.2016.01.012

11. Passlick B, Flieger D, Ziegler-Heitbrock L. Identification and characterization of a novel monocyte subpopulation in human peripheral blood. Blood. 1989;74: 2527-2534. Available: www.bloodjournal.org

12. Ziegler-Heitbrock L, Ancuta P, Crowe S, Dalod M, Grau V, Hart DN, et al. Nomenclature of monocytes and dendritic cells in blood [Internet]. Blood. 2010. doi:10.1182/blood-2010-02-258558

13. Patel AA, Zhang Y, Fullerton JN, Boelen L, Rongvaux A, Maini AA, et al. The fate and lifespan of human monocyte subsets in steady state and systemic inflammation. J Exp Med. 2017;214: 1913-1923. doi:10.1084/jem.20170355

14. Wong KL, Jing-Yi Tai J, Wong W-C, Han H, Sem X, Yeap W-H, et al. Gene expression profiling reveals the defining features of the classical, intermediate, and nonclassical human monocyte subsets New official nomenclature subdivides human monocytes into 3 subsets: the classical (CD14 CD16), intermediate (CD14 CD16 ), and. Blood. 2011;118. doi:10.1182/blood-2010 
15. Yasaka T, Manitich NM, Boxer LA, Baehner RL. Functions of human monocyte and lymphocyte subsets obtained by countercurrent centrifugal elutriation: Differing functional capacities of human monocyte subsets. J Immunol. 1981;127: 15151518.

16. Kurihara T, Warr G, Loy J, Bravo R. Defects in macrophage recruitment and host defense in mice lacking the CCR2 chemokine receptor. J Exp Med. 1997;186: 1757-1762. doi:10.1084/jem.186.10.1757

17. Shi C, Pamer EG. Monocyte recruitment during infection and inflammation. Nature Reviews Immunology. 2011. pp. 762-774. doi:10.1038/nri3070

18. Yang J, Zhang L, Yu C, Yang XF, Wang H. Monocyte and macrophage differentiation: Circulation inflammatory monocyte as biomarker for inflammatory diseases. Biomarker Research. 2014. doi:10.1186/2050-7771-2-1

19. Cros J, Cagnard N, Woollard K, Patey N, Zhang SY, Senechal B, et al. Human CD14dim Monocytes Patrol and Sense Nucleic Acids and Viruses via TLR7 and TLR8 Receptors. Immunity. 2010;33: 375-386. doi:10.1016/j.immuni.2010.08.012

20. Kwissa M, Nakaya HI, Onlamoon N, Wrammert J, Villinger F, Perng GC, et al. Dengue virus infection induces expansion of a CD14+CD16 + monocyte population that stimulates plasmablast differentiation. Cell Host Microbe. 2014;16: 115-127. doi:10.1016/j.chom.2014.06.001

21. Appleby LJ, Nausch N, Midzi N, Mduluza T, Allen JE, Mutapi F. Sources of heterogeneity in human monocyte subsets. Immunol Lett. 2013;152: 32-41. doi:10.1016/j.imlet.2013.03.004

22. Thieblemont N, Weiss L, Sadeghi HM, Estcourt C, Haeffner-Cavaillon N. CD14lowCD16high: A cytokine-producing monocyte subset which expands during human immunodeficiency virus infection. Eur J Immunol. 1995;25: 3418-3424. doi:10.1002/eji.1830251232

23. Michlmayr D, Pak TR, Rahman AH, Amir E-AD, Kim E-Y, Kim-Schulze S, et al. Comprehensive innate immune profiling of chikungunya virus infection in pediatric cases. Mol Syst Biol. 2018;14: e7862. doi:10.15252/msb.20177862 
24. Diebold SS, Kaisho T, Hemmi H, Akira S, Reis E Sousa C. Innate Antiviral Responses by Means of TLR7-Mediated Recognition of Single-Stranded RNA. Science (80- ). 2004;303: 1529-1531. doi:10.1126/science.1093616

25. Heil F, Hemmi H, Hochrein H, Ampenberger F, Kirschning C, Akira S, et al. SpeciesSpecific Recognition of Single-Stranded RNA via Till-like Receptor 7 and 8. Science (80- ). 2004;303: 1526-1529. doi:10.1126/science.1093620

26. Ellery PJ, Tippett E, Chiu Y-L, Paukovics G, Cameron PU, Solomon A, et al. The CD16 + Monocyte Subset Is More Permissive to Infection and Preferentially Harbors HIV-1 In Vivo . J Immunol. 2007;178: 6581-6589. doi:10.4049/jimmunol.178.10.6581

27. Coquillard G, Patterson BK. Determination of Hepatitis C Virus-Infected, Monocyte Lineage Reservoirs in Individuals With or Without HIV Coinfection. J Infect Dis. 2009;200: 947-954. doi:10.1086/605476

28. Rodríguez-Muñoz Y, Martín-Vílchez S, Lõpez-Rodríguez R, Hernández-Bartolomé Á, Trapero-Marugán M, Borque MJ, et al. Peripheral blood monocyte subsets predict antiviral response in chronic hepatitis C. Aliment Pharmacol Ther. 2011;34: 960-971. doi:10.1111/j.1365-2036.2011.04807.x

29. Michlmayr D, Andrade P, Gonzalez K, Balmaseda A, Harris E. CD14(+)CD16(+) monocytes are the main target of Zika virus infection in peripheral blood mononuclear cells in a paediatric study in Nicaragua. Nat Microbiol. 2017; doi:10.1038/s41564-017-0035-0

30. Kawanaka N, Yamamura M, Aita T, Morita Y, Okamoto A, Kawashima M, et al. CD14+,CD16+ blood monocytes and joint inflammation in rheumatoid arthritis. Arthritis Rheum. 2002;46: 2578-2586. doi:10.1002/art.10545

31. Rossol M, Kraus S, Pierer M, Baerwald C, Wagner U. The CD14 brightCD16+ monocyte subset is expanded in rheumatoid arthritis and promotes expansion of the Th17 cell population. Arthritis Rheum. 2012;64: 671-677. doi:10.1002/art.33418

32. Iwahashi M, Yamamura M, Aita T, Okamoto A, Ueno A, Ogawa N, et al. Expression of Toll-Like Receptor 2 on CD16+ Blood Monocytes and Synovial Tissue 
Macrophages in Rheumatoid Arthritis. Arthritis Rheum. 2004;50: 1457-1467. doi:10.1002/art.20219

33. Tsukamoto M, Seta N, Yoshimoto K, Suzuki K, Yamaoka K, Takeuchi T. CD14brightCD16+ intermediate monocytes are induced by interleukin-10 and positively correlate with disease activity in rheumatoid arthritis. Arthritis Res Ther. 2017;19: 28. doi:10.1186/s13075-016-1216-6

34. Chimen M, Yates CM, McGettrick HM, Ward LSC, Harrison MJ, Apta B, et al. Monocyte Subsets Coregulate Inflammatory Responses by Integrated Signaling through TNF and IL-6 at the Endothelial Cell Interface. J Immunol. 2017;198: 2834-2843. doi:10.4049/jimmunol.1601281

35. Dayyani F, Belge K-U, Frankenberger M, Mack M, Berki T, Ziegler-Heitbrock L. Mechanism of glucocorticoid-induced depletion of human CD14 + CD16 + monocytes . J Leukoc Biol. 2003;74: 33-39. doi:10.1189/jlb.1202612

36. Sunderkötter C, Nikolic T, Dillon MJ, van Rooijen N, Stehling M, Drevets DA, et al. Subpopulations of Mouse Blood Monocytes Differ in Maturation Stage and Inflammatory Response. J Immunol. 2004;172: 4410-4417. doi:10.4049/jimmunol.172.7.4410

37. Yrlid U, Jenkins CD, MacPherson GG. Relationships between Distinct Blood Monocyte Subsets and Migrating Intestinal Lymph Dendritic Cells In Vivo under Steady-State Conditions. J Immunol. 2006;176: 4155-4162. doi:10.4049/jimmunol.176.7.4155

38. Yona S, Kim KW, Wolf Y, Mildner A, Varol D, Breker M, et al. Fate Mapping Reveals Origins and Dynamics of Monocytes and Tissue Macrophages under Homeostasis. Immunity. 2013;38: 79-91. doi:10.1016/j.immuni.2012.12.001

39. Thomas GD, Hanna RN, Vasudevan NT, Hamers AA, Romanoski CE, McArdle S, et al. Deleting an Nr4a1 Super-Enhancer Subdomain Ablates Ly6Clow Monocytes while Preserving Macrophage Gene Function. Immunity. 2016;45: 975-987. doi:10.1016/j.immuni.2016.10.011

40. Neighbours LM, Long K, Whitmore AC, Heise MT. Myd88-Dependent Toll-Like Receptor 7 Signaling Mediates Protection from Severe Ross River Virus-Induced 
Disease in Mice. J Virol. 2012;86: 10675-10685. doi:10.1128/jvi.00601-12

654

655

656

657

658

659

660

661

662

663

664

665

666

667

668

669

670

671

672

673

674

675

676

677

678

679

680

681

682

41. Paul AM, Acharya D, Le L, Wang P, Stokic DS, Leis AA, et al. TLR8 Couples SOCS-1 and Restrains TLR7-Mediated Antiviral Immunity, Exacerbating West Nile Virus Infection in Mice. J Immunol. 2016;197: 4425-4435. doi:10.4049/jimmunol.1600902

42. Her Z, Teng T, Tan JJ, Teo T, Kam Y, Lum F, et al. Loss of TLR3 aggravates CHIKV replication and pathology due to an altered virus-specific neutralizing antibody response. EMBO Mol Med. 2015;7: 24-41. doi:10.15252/emmm.201404459

43. Sanchez David RY, Combredet C, Sismeiro O, Dillies MA, Jagla B, Coppée JY, et al. Comparative analysis of viral RNA signatures on different RIG-I-like receptors. Elife. 2016;5. doi:10.7554/eLife.11275

44. Mora E, Guglielmotti A, Biondi G, Sassone-Corsi P. Bindarit: An anti-inflammatory small molecule that modulates the NFKB pathway. Cell Cycle. 2012;11: 159-169. doi:10.4161/cc.11.1.18559

45. Chen W, Foo S-S, Taylor A, Lulla A, Merits A, Hueston L, et al. Bindarit, an Inhibitor of Monocyte Chemotactic Protein Synthesis, Protects against Bone Loss Induced by Chikungunya Virus Infection. J Virol. 2015;89: 581-593.

doi:10.1128/jvi.02034-14

46. Simmons G, Brès V, Lu K, Liss NM, Brambilla DJ, Ryff KR, et al. High incidence of chikungunya virus and frequency of viremic blood donations during epidemic, Puerto Rico, USA, 2014. Emerg Infect Dis. 2016;22: 1221-1228. doi:10.3201/eid2207.160116

47. Pu D, Wang W. Toll-like receptor 4 agonist, lipopolysaccharide, increases the expression levels of cytokines and chemokines in human peripheral blood mononuclear cells. Exp Ther Med. 2014;8: 1914-1918. doi:10.3892/etm.2014.2025

48. van de Weg CAM, Pannuti CS, de Araújo ESA, van den Ham H-J, Andeweg AC, Boas LS V., et al. Microbial Translocation Is Associated with Extensive Immune Activation in Dengue Virus Infected Patients with Severe Disease. Rico-Hesse R, editor. PLoS Negl Trop Dis. 2013;7: e2236. doi:10.1371/journal.pntd.0002236 
49. van de Weg CAM, Koraka P, van Gorp ECM, Mairuhu ATA, Supriatna M, Soemantri A, et al. Lipopolysaccharide levels are elevated in dengue virus infected patients and correlate with disease severity. J Clin Virol. 2012;53: 38-42. doi:10.1016/j.jcv.2011.09.028

50. Klatt NR, Funderburg NT, Brenchley JM. Microbial translocation, immune activation, and HIV disease. Trends in Microbiology. 2013. doi:10.1016/j.tim.2012.09.001

51. Baronti C, Pastorino B, Charrel R, de Lamballerie X. Mycoplasma removal: Simple curative methods for viral supernatants. J Virol Methods. 2013;187: 234-237. doi:10.1016/j.jviromet.2012.09.014

52. Pohjala L, Utt A, Varjak M, Lulla A, Merits A, Ahola T, et al. Inhibitors of alphavirus entry and replication identified with a stable Chikungunya replicon cell line and virus-based assays. PLoS One. 2011;6: e28923.

doi:10.1371/journal.pone.0028923

53. Van Duijl-Richter MKS, Blijleven JS, van Oijen AM, Smit JM. Chikungunya virus fusion properties elucidated by single-particle and bulk approaches. J Gen Virol. 2015;96: 2122-2132. doi:10.1099/vir.0.000144

54. Kurniati NF, Jongman RM, Vom Hagen F, Spokes KC, Moser J, Regan ER, et al. The flow dependency of Tie2 expression in endotoxemia. Intensive Care Med. 2013;39: 1262-1271. doi:10.1007/s00134-013-2899-7 